properties. Many salts of thallium are also photosensitive and have found use in this way to a limited extent. Since its discovery about 100 years ago, the chemistry of thallium has become fairly well known and there are numerous methods available for its determination, both classical and instrumental. In this work an unusually full account is given of the qualitative reactions of thallium (43 pages) with many drawings of organic reagents and some excellent photomicrographs showing crystal developments. This is followed by a brief account of the position of thallium within the classical group separation scheme, and quantitative separation methods based on procipitation, co-precipitation and liquid-liquid distribution are fully described. In the quantitative analysis section, gravimetric and titrimetric methods are discussed very adequately in an economic way and a fine account is given of electrochemical and optical (chiefly solution) methods of determination. The book ends with an extensive series of tables giving a very good account of the physical and chemical constants of a wide range of thallium compounds. There are more than 900 references and a good subject index. On the whole this is an attractive and worthwhile, if somewhat classical, account of the analytical chemistry of thallium which is written in a concise and eminently readable style. While one would have, perhaps, liked to see more attention given to more recent techniques which do not even receive mention and yet are very applicable to the analytical study of thallium, for example, atomic absorption and fluorescence, selective ion electrode methods and the like, one cannot really criticize the book for this, but rather express regret that the author did not go on to extend his account to these topics also.

Analytical Chemistry of Cobalt. Cobalt has been a familiar element to man since the times of ancient Egypt and the dynastic periods of China where it was used extensively as a blue pigment. The element itself has numerous uses in alloys because of the desirable properties it gives to steel, and the like, and the recent introduction of cobalt-nickel based high temperature alloys for aeroturbines has intensified interest in the element in recent years. This volume first of all discusses the analytical characteristics of cobalt compounds by describing the chemical and physical behaviour of most of the simple salts of the metal and many of its complex compounds with other inorganic ions and with organic molecules. This is a very full and quite unusual account, rarely seen in most analy tical textbooks. There follows an account of methods for the detection and identification of cobalt which is perhaps much fuller than need be in view of the array of modern methods now available to replace those largely classical procedures. A separate chapter details separation/ concentration methods for cobalt in the presence of other metals. This account covers precipitation, co-precipita. tion, solvent extraction, adsorption and ion-exchange chromatography, electrophoresis and so on. A full account is given of the gravimetric, titrimetric and colorimetric determination of cobalt using (chiefly) organic reagents. The ensuing discussion of polarographic, catalytic and activation methods is, on the other hand, rather too brief. The final chapter deals with the determination of cobalt in ores, slags, silicates, steel and otherferrous alloys, non-ferrous alloys, soils, plants, animal tissues, and the like. On the whole, this volume in the series is cast in rather too classical a manner. References are made in a very few instances near the end of the first chapter to the spectroscopic analysis of cobalt following isolation, but no details are given and a simple (solution) photometric procedure is usually recommended or a polarographic method. In many instances much less involved procedures are available by use of more modern techniques, for example, atomic absorption which was certainly available in 1963 when the author compiled his review. Although it is excellent in its own way, I think this volume gives rather too classical a slant to its account of the analytical chemistry of cobalt.

T. S. WEST

\section{CARBOXYLIC ACID CHEMISTRY} $\mathrm{COOH}(\mathrm{R})$ : The Chemistry of Carboxylic Acids and Esters

(The Chemistry of Functional Groups.) Edited by Saul Patai. Pp. xiv +1155 . (Wiley (Interscience): New York and London, 1969.) $300 s$.

THIs is the sixth of the compendia treating a single functional group published by Interscience under the general editorship of S. Patai. Both the price and the scope of the contents indicate that these volumes are destined solely for library purchase; it seems unlikely that private individuals will be prepared to pay $£ 15$ for subjects so diverse as theoretical aspects of the $\mathrm{COOH}$ and COOR groups, and their analytical chemistry. At approximately $3 d$ a page, however, the book is not overpriced.

One object of the series is to present review articles on subjects which have not been treated elsewhere. In some cases this policy produces a very useful review; for example, chapter four on the formation of carboxylic acids from organometallic compounds is very welcome. On the other hand, there are notable failures. Chapter twelve on decarboxylation contains only a single reference after 1954 to the original literature of decarboxylation (1963), other than the author's own rather esoteric contributions. Further, it is difficult to see how the long (45 pages) chapter on the analysis of carboxylic acids can be expected to show any novelty. This chapter adds very little to the book; it is a collection of chemical and physical methods which can be found readily elsewhere, and it is difficult to understand why the author should think it necessary to spend a little more than 4 pages in a book at this level in telling the reader how to titrate an acid with alkali, and how to present and work out his results.

The book, consisting of contributions from eighteen different sources, is bound to be variable in standard. The authors of the first chapter ("General and Theoretical Aspects of the COOH and COOR Groups") are to be congratulated for presenting their excellent material at a level comprehensible to most organic chemists. It is followed by a useful review of the Kolbe reaction. Chapter thirteen presents an interesting review of the chemistry of ortho esters, discussing both reactions and mechanism, but the following chapter on peracids and peresters disappoints in its paucity of mechanistic discussion. Perhaps the most interesting chapter to the organic chemist is the last one, on the biological formation and reactions of the $-\mathrm{COOH}$ and $-\mathrm{COOR}$ groups. Once the formidable task of memorizing the biochemical abbreviations has been performed (helped by an alphabetical list at the beginning of the chapter), this long chapter is a mine of fascinating information.

Finally, the book seems to have had a rather long gestation period. Published in 1969 , it does not seem to contain references beyond 1965-66, at least in number.

D. BIGLEY

\section{PROTEIN STRUCTURE}

\section{The Structure and Action of Proteins}

By Richard E. Dickerson and Irving Geis. Pp. vii +119. (Harper and Row: New York and London, 1969.) \$4.95.

From the outset this book emphasizes the evolution and the real physical scale of proteins. From the composition of living systems, it progresses to linear protein polymers and their constituent amino-acids, discussing, too, the intrinsic properties of the polypeptide backbone, the side chain functional groups and the optical activity of the monomers. The linear trinucleotide genetic code and the chemical properties of the amino-acids are elegantly related and the structural proteins are clearly described. There is a satisfying chapter on the oxygen carrying molocules and then a stimulating discussion on 